\title{
Discriminative Random Fields: A Discriminative Framework for Contextual Interaction in Classification
}

\author{
Sanjiv Kumar and Martial Hebert \\ The Robotics Institute, Carnegie Mellon University \\ Pittsburgh, PA 15213, USA, \{skumar, hebert $\} @$ ri.cmu.edu
}

\begin{abstract}
In this work we present Discriminative Random Fields (DRFs), a discriminative framework for the classification of image regions by incorporating neighborhood interactions in the labels as well as the observed data. The discriminative random fields offer several advantages over the conventional Markov Random Field (MRF) framework. First, the DRFs allow to relax the strong assumption of conditional independence of the observed data generally used in the MRF framework for tractability. This assumption is too restrictive for a large number of applications in vision. Second, the DRFs derive their classification power by exploiting the probabilistic discriminative models instead of the generative models used in the MRF framework. Finally, all the parameters in the DRF model are estimated simultaneously from the training data unlike the MRF framework where likelihood parameters are usually learned separately from the field parameters. We illustrate the advantages of the DRFs over the MRF framework in an application of man-made structure detection in natural images taken from the Corel database.
\end{abstract}

\section{Introduction}

The problem of region classification, i.e. segmentation and labeling of image regions is of fundamental interest in computer vision. For the analysis of natural images, it is important to use the contextual information in the form of spatial dependencies in the images. Markov Random Field (MRF) models have been used extensively for various segmentation and labeling applications in vision, which allow one to incorporate contextual constraints in a principled manner [15].

MRFs are generally used in a probabilistic generative framework that models the joint probability of the observed data and the corresponding labels. In other words, let $\boldsymbol{y}$ be the observed data from an input image, where $\boldsymbol{y}=\left\{\boldsymbol{y}_{i}\right\}_{i \in S}$, $\boldsymbol{y}_{i}$ is the data from the $i^{\text {th }}$ site, and $S$ is the set of sites.
Let the corresponding labels at the image sites be given by $\boldsymbol{x}=\left\{x_{i}\right\}_{i \in S}$. In the MRF framework, the posterior over the labels given the data is expressed using the Bayes' rule as,

$$
P(\boldsymbol{x} \mid \boldsymbol{y}) \propto p(\boldsymbol{x}, \boldsymbol{y})=P(\boldsymbol{x}) p(\boldsymbol{y} \mid \boldsymbol{x})
$$

where the prior over labels, $P(\boldsymbol{x})$ is modeled as a MRF. For computational tractability, the observation or likelihood model, $p(\boldsymbol{y} \mid \boldsymbol{x})$ is assumed to have a factorized form, i.e. $p(\boldsymbol{y} \mid \boldsymbol{x})=\prod_{i \in S} p\left(\boldsymbol{y}_{i} \mid x_{i}\right)$ [1][4][15][22]. However, as noted by several researchers [2][13][18][20], this assumption is too restrictive for several applications in vision. For example, consider a class that contains man-made structures (e.g. buildings). The data belonging to such a class is highly dependent on its neighbors. This is because, in man-made structures, the lines or edges at spatially adjoining sites follow some underlying organization rules rather than being random (See Figure 1 (a)). This is also true for a large number of texture classes that are made of structured patterns. In this work we have chosen the application of man-made structure detection purely as a source of data to show the advantages of the Discriminative Random Field (DRF) model.

Some efforts have been made in the past to model the dependencies in the data. In [11], a technique has been presented that assumes the noise in the data at neighboring sites to be correlated, which is modeled using an auto-normal model. However, the authors do not specify a field over the labels and classify a site by maximizing the local posterior over labels given the data and the neighborhood labels. In probabilistic relaxation labeling, either the labels are assumed to be independent given the relational measurements at two or more sites [3] or conditionally independent in local neighborhood of a site given its label [10]. In the context of hierarchical texture segmentation, Won and Derin [21] model the local joint distribution of the data contained in the neighborhood of a site assuming all the neighbors from the same class. They further approximate the overall likelihood to be factored over the local joint distributions. Wilson and $\mathrm{Li} \mathrm{[20]} \mathrm{assume} \mathrm{the} \mathrm{difference} \mathrm{between} \mathrm{observations}$ from the neighboring sites to be conditionally independent 
given the label field.

In the context of multiscale random field, Cheng and Bouman [2] make a more general assumption. They assume the difference between the data at a given site and the linear combination of the data from that site's parents to be conditionally independent given the label at the current scale. All the above techniques make simplifying assumptions to get some sort of factored approximation of the likelihood for tractability. This precludes capturing stronger relationships in the observations in the form of arbitrarily complex features that might be desired to discriminate between different classes. A novel pairwise MRF model is suggested in [18] to avoid the problem of explicit modeling of the likelihood, $p(\boldsymbol{y} \mid \boldsymbol{x})$. They model the joint $p(\boldsymbol{x}, \boldsymbol{y})$ as a MRF in which the label field $P(\boldsymbol{x})$ is not necessarily a MRF. But this shifts the problem to the modeling of pairs $(\boldsymbol{x}, \boldsymbol{y})$. The authors model the pair by assuming the observations to be the true underlying binary field corrupted by correlated noise. However, for most of the real-world applications, this assumption is too simplistic. In our previous work [13], we modeled the data dependencies using a pseudolikelihood approximation of a conditional MRF for computational tractability. In this work, we explore alternative ways of modeling data dependencies which permit eliminating these approximations in a principled manner.

Now considering a different point of view, for classification purposes, we are interested in estimating the posterior over labels given the observations, i.e., $P(\boldsymbol{x} \mid \boldsymbol{y})$. In a generative framework, one expends efforts to model the joint distribution $p(\boldsymbol{x}, \boldsymbol{y})$, which involves implicit modeling of the observations. In a discriminative framework, one models the distribution $P(\boldsymbol{x} \mid \boldsymbol{y})$ directly. As noted in [4], a potential advantage of using the discriminative approach is that the true underlying generative model may be quite complex even though the class posterior is simple. This means that the generative approach may spend a lot of resources on modeling the generative models which are not particularly relevant to the task of inferring the class labels. Moreover, learning the class density models may become even harder when the training data is limited [19].

In this work we present a new model called Discriminative Random Field based on the concept of Conditional Random Field (CRF) proposed by Lafferty et al. [14] in the context of segmentation and labeling of the 1-D text sequences. The CRFs directly model the posterior distribution $P(\boldsymbol{x} \mid \boldsymbol{y})$ as a Gibbs field. This approach allows one to capture arbitrary dependencies between the observations without resorting to any model approximations. CRFs have been shown to outperform the traditional Hidden Markov Model based labeling of text sequences [14]. Our model further enhances the CRFs by proposing the use of local discriminative models to capture the class associations at individual sites as well as the interactions with the neighboring sites on

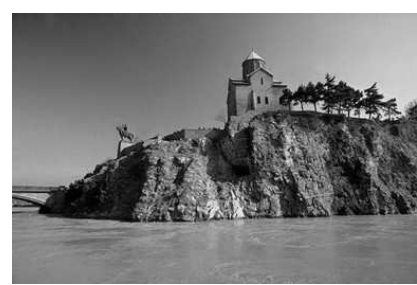

(a) Input image

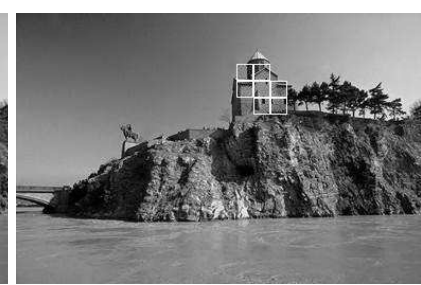

(b) DRF result
Figure 1. A natural image and the corresponding DRF result. A bounding square indicates the presence of structure at that block. This example is to illustrate the fact that modeling data dependency is important for the detection of man-made structures.

2-D lattices. The proposed DRF model permits interactions in both the observed data and the labels. An example result of the DRF model applied to man-made structure detection is shown in Figure 1 (b).

\section{Discriminative Random Field}

We first restate in our notations the definition of the Conditional Random Fields as given by Lafferty et al. [14]. As defined before, the observed data from an input image is given by $\boldsymbol{y}=\left\{\boldsymbol{y}_{i}\right\}_{i \in S}$ where $\boldsymbol{y}_{i}$ is the data from $i^{\text {th }}$ site and $\boldsymbol{y}_{i} \in \Re^{c}$. The corresponding labels at the image sites are given by $\boldsymbol{x}=\left\{x_{i}\right\}_{i \in S}$. In this work we will be concerned with binary classification, i.e. $x_{i} \in\{-1,1\}$. The random variables $\boldsymbol{x}$ and $\boldsymbol{y}$ are jointly distributed, but in a discriminative framework, a conditional model $P(\boldsymbol{x} \mid \boldsymbol{y})$ is constructed from the observations and labels, and the marginal $p(\boldsymbol{y})$ is not modeled explicitly.

CRF Definition: Let $G=(S, E)$ be a graph such that $\boldsymbol{x}$ is indexed by the vertices of $G$. Then $(\boldsymbol{x}, \boldsymbol{y})$ is said to be a conditional random field if, when conditioned on $\boldsymbol{y}$, the random variables $x_{i}$ obey the Markov property with respect to the graph: $P\left(x_{i} \mid \boldsymbol{y}, \boldsymbol{x}_{S-\{i\}}\right)=P\left(x_{i} \mid \boldsymbol{y}, \boldsymbol{x}_{\mathcal{N}_{i}}\right)$, where $S-\{i\}$ is the set of all nodes in the graph except the node $i, \mathcal{N}_{i}$ is the set of neighbors of the node $i$ in $G$, and $\boldsymbol{x}_{\Omega}$ represents the set of labels at the nodes in set $\Omega$.

Thus, a CRF is a random field globally conditioned on the observations $\boldsymbol{y}$. The condition of positivity requiring $P(\boldsymbol{x} \mid \boldsymbol{y})>0 \forall \boldsymbol{x}$ has been assumed implicitly. Now, using the Hammersley Clifford theorem [15] and assuming only up to pairwise clique potentials to be nonzero, the joint distribution over the labels $\boldsymbol{x}$ given the observations $\boldsymbol{y}$ can be written as,

$P(\boldsymbol{x} \mid \boldsymbol{y})=\frac{1}{Z} \exp \left(\sum_{i \in S} A_{i}\left(x_{i}, \boldsymbol{y}\right)+\sum_{i \in S} \sum_{j \in \mathcal{N}_{i}} I_{i j}\left(x_{i}, x_{j}, \boldsymbol{y}\right)\right)$

where $Z$ is a normalizing constant known as the partition 
function, and $-A_{i}$ and $-I_{i j}$ are the unary and pairwise potentials respectively. With a slight abuse of notations, in the rest of the paper we will call $A_{i}$ the association potential and $I_{i j}$ the interaction potential. Note that both terms explicitly depend on all the observations $\boldsymbol{y}$. Lafferty et al. [14] modeled the association and the interaction potentials as linear combinations of a predefined set of features from text sequences. In contrast, we look at the association potential as a local decision term which decides the association of a given site to a certain class ignoring its neighbors. In the MRF framework, with the assumption of conditional independence of the data, this potential is similar to the log likelihood of the data at that site. The interaction potential is seen in DRFs as a data dependent smoothing function. In the rest of the paper we assume the random field given in Eq. (1) to be homogeneous and isotropic, i.e. the functional forms of $A_{i}$ and $I_{i j}$ are independent of the locations $i$ and $j$. Henceforth we will leave the subscripts and simply use the notations $A$ and $I$. Note that the assumption of isotropy can be easily relaxed at the cost of a few additional parameters.

\subsection{Association Potential}

In the DRF framework, $A\left(x_{i}, \boldsymbol{y}\right)$ is modeled using a local discriminative model that outputs the association of the site $i$ with class $x_{i}$. Generalized Linear Models (GLM) are used extensively in statistics to model the class posteriors given the observations [16]. For each site $i$, let $\boldsymbol{f}_{i}(\boldsymbol{y})$ be a function that maps the observations $\boldsymbol{y}$ on a feature vector such that $\boldsymbol{f}_{i}: \boldsymbol{y} \rightarrow \Re^{l}$. Using the logistic function as the link, the local class posterior can be modeled as,

$$
P\left(x_{i}=1 \mid \boldsymbol{y}\right)=\frac{1}{1+e^{-\left(w_{0}+\boldsymbol{w}_{1}^{T} \boldsymbol{f}_{i}(\boldsymbol{y})\right)}}=\sigma\left(w_{0}+\boldsymbol{w}_{1}^{T} \boldsymbol{f}_{i}(\boldsymbol{y})\right)
$$

where $\boldsymbol{w}=\left\{w_{0}, \boldsymbol{w}_{1}\right\}$ are the model parameters. To extend the logistic model to induce a nonlinear decision boundary in the feature space, a transformed feature vector at each site $i$ is defined as, $\boldsymbol{h}_{i}(\boldsymbol{y})=\left[1, \phi_{1}\left(\boldsymbol{f}_{i}(\boldsymbol{y})\right), \ldots, \phi_{R}\left(\boldsymbol{f}_{i}(\boldsymbol{y})\right)\right]^{T}$ where $\phi_{k}($.$) are arbitrary nonlinear functions. The first ele-$ ment of the transformed vector is kept as 1 to accommodate the bias parameter $w_{0}$. Further, since $x_{i} \in\{-1,1\}$, the probability in Eq. (2) can be compactly expressed as,

$$
P\left(x_{i} \mid \boldsymbol{y}\right)=\sigma\left(x_{i} \boldsymbol{w}^{T} \boldsymbol{h}_{i}(\boldsymbol{y})\right)
$$

Finally, the association potential is defined as,

$$
A\left(x_{i}, \boldsymbol{y}\right)=\log \left(\sigma\left(x_{i} \boldsymbol{w}^{T} \boldsymbol{h}_{i}(\boldsymbol{y})\right)\right)
$$

This transformation ensures that the DRF is equivalent to a logistic classifier if the interaction potential in Eq. (1) is set to zero. Note that the transformed feature vector at $e a c h$ site $i$, i.e. $\boldsymbol{h}_{i}(\boldsymbol{y})$ is a function of whole set of observations $\boldsymbol{y}$. On the contrary, the assumption of conditional independence of the data in the MRF framework allows one to use the data only from a particular site, i.e. $\boldsymbol{y}_{i}$ to get the log-likelihood, which acts as the association potential.

As a related work, in the context of tree-structured belief networks, Feng et al. [4] used the scaled likelihoods to approximate the actual likelihoods at each site required by the generative formulation. These scaled likelihoods were obtained by scaling the local class posteriors learned using a neural network. On the contrary, in the DRF model, the local class posterior is an integral part of the full conditional model in Eq. (1).

\subsection{Interaction Potential}

To model the interaction potential, $I$, we first analyze the form commonly used in the MRF framework. For the isotropic, homogeneous Ising model, the interaction potential is given as $I=\beta x_{i} x_{j}$, which penalizes every dissimilar pair of labels by the cost $\beta$ [15]. This form of interaction favors piecewise constant smoothing of the labels without considering the discontinuities in the observed data explicitly. Geman and Geman [7] have proposed a line-process model which allows discontinuities in the labels to provide piecewise continuous smoothing. Other discontinuity models have also been proposed for adaptive smoothing [15], but all of them are independent of the observed data. In the DRF formulation, the interaction potential is a function of all the observations $\boldsymbol{y}$. We propose to model $I$ in DRFs using a data-dependent term along with the constant smoothing term of the Ising model. In addition to modeling arbitrary pairwise relational information between sites, the data-dependent smoothing can compensate for the errors in modeling the association potential. To model the data-dependent term, the aim is to have similar labels at a pair of sites for which the observed data supports such a hypothesis. In other words, we are interested in learning a pairwise discriminative model $p\left(x_{i}=x_{j} \mid \boldsymbol{\psi}_{i}(\boldsymbol{y}), \boldsymbol{\psi}_{j}(\boldsymbol{y})\right)$ where $\boldsymbol{\psi}_{k}: \boldsymbol{y} \rightarrow \Re^{\gamma}$. Note that by choosing the function $\boldsymbol{\psi}_{i}$ to be different from $\boldsymbol{f}_{i}$, used in Eq.(2), information different from $\boldsymbol{f}_{i}$ can be used to model the relations between pairs of sites.

Let $t_{i j}$ be an auxiliary variable defined as,

$$
t_{i j}= \begin{cases}+1 & \text { if } x_{i}=x_{j} \\ -1 & \text { otherwise }\end{cases}
$$

and let $\boldsymbol{\mu}_{i j}\left(\boldsymbol{\psi}_{i}(\boldsymbol{y}), \boldsymbol{\psi}_{j}(\boldsymbol{y})\right)$ be a new feature vector such that $\boldsymbol{\mu}_{i j}: \Re^{\gamma} \times \Re^{\gamma} \rightarrow \Re^{q}$. Denoting this feature vector as $\boldsymbol{\mu}_{i j}(\boldsymbol{y})$ for simplification, we model the pairwise discriminatory term similar to the one defined in Eq.(3) as,

$$
P\left(t_{i j} \mid \boldsymbol{\psi}_{i}(\boldsymbol{y}), \boldsymbol{\psi}_{j}(\boldsymbol{y})\right)=\sigma\left(t_{i j} \boldsymbol{v}^{T} \boldsymbol{\mu}_{i j}(\boldsymbol{y})\right)
$$

Where $v$ are the model parameters. Note that the first component of $\boldsymbol{\mu}_{i j}(\boldsymbol{y})$ is fixed to be 1 to accommodate the bias 
parameter. Now, the interaction potential in DRFs is modeled as a convex combination of two terms, i.e.

$$
\begin{aligned}
I\left(x_{i}, x_{j}, \boldsymbol{y}\right)= & \beta\left\{K x_{i} x_{j}\right. \\
& \left.+(1-K)\left(2 \sigma\left(t_{i j} \boldsymbol{v}^{T} \boldsymbol{\mu}_{i j}(\boldsymbol{y})\right)-1\right)\right\}(6)
\end{aligned}
$$

where $0 \leq K \leq 1$. The first term is a data-independent smoothing term, similar to the Ising model. The second term is a $[-1,1]$ mapping of the pairwise logistic function defined in Eq. (5). This mapping ensures that both terms have the same range. Ideally, the data-dependent term will act as a discontinuity adaptive model that will moderate the smoothing when the data from two sites is 'different'. The parameter $K$ gives the flexibility to the model by allowing the learning algorithm to adjust the relative contributions of these two terms according to the training data. Finally, $\beta$ is the interaction coefficient that controls the degree of smoothing. Large values of $\beta$ encourage more smooth solutions. Note that even though the model seems to have some resemblance to the line process suggested in [7], $K$ in Eq. (6) is a global weighting parameter unlike the line process where a discrete parameter is introduced for each pair of sites to facilitate discontinuities in smoothing. Anisotropy can be easily included in the DRF model by parametrizing the interaction potentials of different directional pairwise cliques with different sets of parameters $\{\beta, K, \boldsymbol{v}\}$.

\section{Parameter Estimation}

Let $\theta$ be the set of parameters of the DRF model where $\theta=\{\boldsymbol{w}, \boldsymbol{v}, \beta, K\}$. The form of the DRF model resembles the posterior for the MRF framework assuming conditionally independent data. However, in the MRF framework, the parameters of the class generative models, $p\left(\boldsymbol{y}_{i} \mid x_{i}\right)$ and the parameters of the prior random field on labels, $P(\boldsymbol{x})$ are generally assumed to be independent and are learned separately [15]. In contrast, we make no such assumption and learn all the parameters of the DRF model simultaneously. Nevertheless, the similarity of the form allows for most of the techniques used for learning the MRF parameters to be utilized for learning the DRF parameters with a few modifications.

We take the standard maximum-likelihood approach to learn the DRF parameters, which involves the evaluation of the partition function $Z$. The evaluation of $Z$ is, in general, a NP-hard problem. One could use either sampling techniques or resort to some approximations e.g. mean-field or pseudolikelihood to estimate the parameters [15]. In this work we used the pseudolikelihood formulation due to its simplicity and consistency of the estimates for the large lattice limit [15]. According to this,

$$
\widehat{\theta}^{M L} \approx \underset{\theta}{\arg \max } \prod_{m=1}^{M} \prod_{i \in S} P\left(x_{i}^{m} \mid \boldsymbol{x}_{\mathcal{N}_{i}}^{m}, \boldsymbol{y}^{m}, \theta\right)
$$

$$
\text { Subject to } 0 \leq K \leq 1
$$

where $m$ indexes over the training images and $M$ is the total number of training images, and

$$
\begin{gathered}
P\left(x_{i} \mid \boldsymbol{x}_{\mathcal{N}_{i}}, \boldsymbol{y}, \theta\right)=\frac{1}{z_{i}} \exp \left\{A\left(x_{i}, \boldsymbol{y}\right)+\sum_{j \in \mathcal{N}_{i}} I\left(x_{i}, x_{j}, \boldsymbol{y}\right)\right\}, \\
z_{i}=\sum_{x_{i} \in\{-1,1\}} \exp \left\{A\left(x_{i}, \boldsymbol{y}\right)+\sum_{j \in \mathcal{N}_{i}} I\left(x_{i}, x_{j}, \boldsymbol{y}\right)\right\}
\end{gathered}
$$

The pseudo-likelihood given in Eq. (7) can be maximized by using line search methods for constrained maximization with bounds [8]. Since the pseudolikelihood is generally not a convex function of the parameters, good initialization of the parameters is important to avoid bad local maxima. To initialize the parameters $\boldsymbol{w}$ in $A\left(x_{i}, \boldsymbol{y}\right)$, we first learn these parameters using standard maximum likelihood logistic regression assuming all the labels $x_{i}^{m}$ to be independent given the data $\boldsymbol{y}^{\boldsymbol{m}}$ for each image $m$ [17]. Using Eq. (3), the log-likelihood can be expressed as,

$$
L(\boldsymbol{w})=\sum_{m=1}^{M} \sum_{i \in S} \log \left(\sigma\left(x_{i}^{m} \boldsymbol{w}^{T} \boldsymbol{h}_{i}\left(\boldsymbol{y}^{\boldsymbol{m}}\right)\right)\right)
$$

The Hessian of the log-likelihood is given as,

$$
\begin{aligned}
\nabla_{\boldsymbol{w}}^{2} L(\boldsymbol{w})= & -\sum_{m=1}^{M} \sum_{i \in S}\left\{\sigma\left(\boldsymbol{w}^{T} \boldsymbol{h}_{i}\left(\boldsymbol{y}^{\boldsymbol{m}}\right)\right)\right. \\
& \left.\left(1-\sigma\left(\boldsymbol{w}^{T} \boldsymbol{h}_{i}\left(\boldsymbol{y}^{\boldsymbol{m}}\right)\right)\right)\right\} \boldsymbol{h}_{i}\left(\boldsymbol{y}^{\boldsymbol{m}}\right) \boldsymbol{h}_{i}^{T}\left(\boldsymbol{y}^{\boldsymbol{m}}\right)
\end{aligned}
$$

Note that the Hessian does not depend on how the data is labeled and is nonpositive definite. Hence the log-likelihood in Eq. (8) is convex, and any local maximum is the global maximum. Newton's method was used for maximization which has been shown to be much faster than other techniques for correlated features [17]. The initial estimates of the parameters $\boldsymbol{v}$ in data-dependent term in $I\left(x_{i}, x_{j}, \boldsymbol{y}\right)$ were also obtained similarly.

\section{Inference}

Given a new test image $\boldsymbol{y}$, our aim is to find the optimal label configuration $\boldsymbol{x}$ over the image sites where optimality is defined with respect to a cost function. Maximum A Posteriori (MAP) solution is a widely used estimate that is optimal with respect to the zero-one cost function defined as $C\left(\boldsymbol{x}, \boldsymbol{x}^{*}\right)=1-\delta\left(\boldsymbol{x}-\boldsymbol{x}^{*}\right)$, where $\boldsymbol{x}^{*}$ is the true label configuration, and $\delta\left(\boldsymbol{x}-\boldsymbol{x}^{*}\right)$ is 1 if $\boldsymbol{x}=\boldsymbol{x}^{*}$, and 0 otherwise. For binary classifications, MAP estimate can be computed exactly using the max-flow/min-cut type of algorithms if the probability distribution meets certain conditions [9][12]. For the DRF model, exact MAP solution can 
be computed if $K \geq 0.5$ and $\beta \geq 0$. However, in the context of MRFs, the MAP solution has been shown to perform poorly for the Ising model when the interaction parameter, $\beta$ takes large values [9][6]. Our results in Section 5.3 corroborate this observation for the DRFs too.

An alternative to the MAP solution is the Maximum Posterior Marginal (MPM) solution for which the cost function is defined as $C\left(\boldsymbol{x}, \boldsymbol{x}^{*}\right)=\sum_{i \in S}\left(1-\delta\left(x_{i}-x_{i}^{*}\right)\right)$, where $x_{i}^{*}$ is the true label at the $i^{t h}$ site. The MPM computation requires marginalization over a large number of variables which is generally NP-hard. One can use either sampling procedures [6] or use Belief Propagation to obtain an estimate of the MPM solution. In this work we chose a simple algorithm, Iterated Conditional Modes (ICM), proposed by Besag [1]. Given an initial label configuration, ICM maximizes the local conditional probabilities iteratively, i.e.

$$
x_{i} \leftarrow \underset{x_{i}}{\arg \max } P\left(x_{i} \mid \boldsymbol{x}_{\mathcal{N}_{i}}, \boldsymbol{y}\right)
$$

ICM yields local maximum of the posterior and has been shown to give reasonably good results even when exact MAP performs poorly for large values of $\beta$ [9][6]. In our ICM implementation, the image sites were divided into coding sets to speed up the sequential updating procedure [1].

\section{Experiments and Discussion}

The proposed DRF model was applied to the task of detecting man-made structures in natural scenes. We have used this application purely as the source of data to show the advantages of the DRF over the MRF framework. The training and the test set contained 108 and 129 images respectively, each of size $256 \times 384$ pixels, from the Corel image database. Each image was divided in nonoverlapping $16 \times 16$ pixels blocks, and we call each such block an image site. The ground truth was generated by hand-labeling every site in each image as a structured or nonstructured block. The whole training set contained 36,269 blocks from the nonstructured class, and 3,004 blocks from the structured class.

\subsection{Feature Description}

The detailed explanation of the features used for the structure detection application is given in [13]. Here we briefly describe the features to set the notations. The intensity gradients contained within a window (defined later) in the image are combined to yield a histogram over gradient orientations. Each histogram count is weighted by the gradient magnitude at that pixel. To alleviate the problem of hard binning of the data, the histogram is smoothed using kernel smoothing. Heaved central-shift moments are computed to capture the the average 'spikeness' of the smoothed histogram as an indicator of the 'structuredness' of the patch. The orientation based feature is obtained by passing the absolute difference between the locations of the two highest peaks of the histogram through sinusoidal nonlinearity. The absolute location of the highest peak is also used.

For each image we compute two different types of feature vectors at each site. Using the same notations as introduced in Section 2, first a single-site feature vector at the site $i, \boldsymbol{s}_{i}\left(\boldsymbol{y}_{\boldsymbol{i}}\right)$ is computed using the histogram from the data $\boldsymbol{y}_{i}$ at that site (i.e., $16 \times 16$ block) such that $\boldsymbol{s}_{i}: \boldsymbol{y}_{\boldsymbol{i}} \rightarrow \Re^{d}$. Obviously, this vector does not take into account influence of the data in the neighborhood of that site. The vector $\boldsymbol{s}_{i}\left(\boldsymbol{y}_{i}\right)$ is composed of first three moments and two orientation based features described above. Next, a multiscale feature vector at the site $i, \boldsymbol{f}_{i}(\boldsymbol{y})$ is computed which explicitly takes into account the dependencies in the data contained in the neighboring sites. It should be noted that the neighborhood for the data interaction need not be the same as for the label interaction. To compute $\boldsymbol{f}_{i}(\boldsymbol{y})$, smoothed histograms are obtained at three different scales, where each scale is defined as a varying window size around the site $i$. The number of scales is chosen to be 3 , with the scales changing in regular octaves. The lowest scale is fixed at $16 \times 16$ pixels (i.e. the size of a single site), and the highest scale at $64 \times 64$ pixels. The moment and orientation based features are obtained at each scale similar to $\boldsymbol{s}_{i}\left(\boldsymbol{y}_{\boldsymbol{i}}\right)$. In addition, two interscale features are also obtained using the highest peaks from the histograms at consecutive scales. To avoid redundancy in the moments based features, only two moment features are used from each scale yielding a 14 dimensional feature vector.

\subsection{Learning}

The parameters of the DRF model $\theta=\{\boldsymbol{w}, \boldsymbol{v}, \beta, K\}$ were learned from the training data using the maximum pseudolikelihood method described in Section 3. For the association potentials, a transformed feature vector $\boldsymbol{h}_{i}(\boldsymbol{y})$ was computed at each site $i$. In this work we used the quadratic transforms such that the functions $\phi_{k}\left(\boldsymbol{f}_{i}(\boldsymbol{y})\right)$ include all the $l$ components of the feature vector $\boldsymbol{f}_{i}(\boldsymbol{y})$, their squares and all the pairwise products yielding $l+l(l+1) / 2$ features [5]. This is equivalent to the kernel mapping of the data using a polynomial kernel of degree two. Any linear classifier in the transformed feature space will induce a quadratic boundary in the original feature space. Since $l$ is 14 , the quadratic mapping gives a 119 dimensional vector at each site. In this work, the function $\psi_{i}$, defined in section 2.2 was chosen to be the same as $\boldsymbol{f}_{i}$. The pairwise data vector $\boldsymbol{\mu}_{i j}(\boldsymbol{y})$ can be obtained either by passing the two vectors $\boldsymbol{\psi}_{i}(\boldsymbol{y})$ and $\boldsymbol{\psi}_{j}(\boldsymbol{y})$ through a distance function, e.g. absolute component wise difference, or by concatenating the two vectors. We used 
the concatenated vector in the present work which yielded slightly better results. This is possibly due to wide within class variations in the nonstructured class. For the interaction potential, first order neighborhood (i.e. four nearest neighbors) was considered similar to the Ising model.

First, the parameters of the logistic functions, $\boldsymbol{w}$ and $\boldsymbol{v}$, were estimated separately to initialize the pseudolikelihood maximization scheme. Newton's method was used for logistic regression and the initial values for all the parameters were set to 0. Since the logistic log-likelihood given in Eq. (8) is convex, initial values are not a concern for the logistic regression. Approximately equal number of data points were used from both classes. For the DRF learning, the interaction parameter $\beta$ was initialized to 0 , i.e. no contextual interaction between the labels. The weighting parameter $K$ was initialized to 0.5 giving equal weights to both the dataindependent and the data-dependent terms in $I\left(x_{i}, x_{j}, \boldsymbol{y}\right)$. All the parameters $\theta$ were learned by using gradient descent for constrained maximization. The final values of $\beta$ and $K$ were found to be 0.77 , and 0.83 respectively. The learning took 100 iterations to converge in $627 \mathrm{~s}$ on a $1.5 \mathrm{GHz}$ Pentium class machine.

To compare the results from the DRF model with those from the MRF framework, we learned the MRF parameters using the pseudolikelihood formulation. The label field $P(\boldsymbol{x})$ was assumed to be a homogeneous and isotropic MRF given by the Ising model with only pairwise nonzero potentials. The data likelihood $p(\boldsymbol{y} \mid \boldsymbol{x})$ was assumed to be conditionally independent given the labels. The posterior for this model is given by,

$P(\boldsymbol{x} \mid \boldsymbol{y})=\frac{1}{Z_{m}} \exp \left(\sum_{i \in S} \log p\left(\boldsymbol{s}_{i}\left(\boldsymbol{y}_{i}\right) \mid x_{i}\right)+\sum_{i \in S} \sum_{j \in \mathcal{N}_{i}} \beta_{m} x_{i} x_{j}\right)$

where $\beta_{m}$ is the interaction parameter of the MRF. Note that $s_{i}\left(\boldsymbol{y}_{i}\right)$ is a single-site feature vector. Each class conditional density was modeled as a mixture of Gaussian. The number of Gaussians in the mixture was selected to be 5 using cross-validation. The mean vectors, full covariance matrices and the mixing parameters were learned using the standard EM technique. The pseudo-likelihood learning algorithm yielded $\beta_{m}$ to be 0.68 . The learning took $9.5 \mathrm{~s}$ to converge in 70 iterations. With a slight abuse of notation, we will use the term MRF to denote the model with above posterior in the rest of the paper.

\subsection{Performance Evaluation}

In this section we present a qualitative as well as a quantitative evaluation of the proposed DRF model. First we compare the detection results on the test images using three different methods: logistic classifier with MAP inference, and MRF and DRF with ICM inference. The ICM algorithm was initialized from the maximum likelihood solution

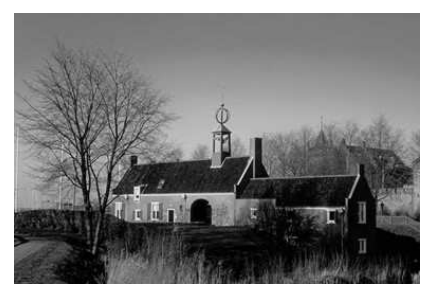

(a) Input image

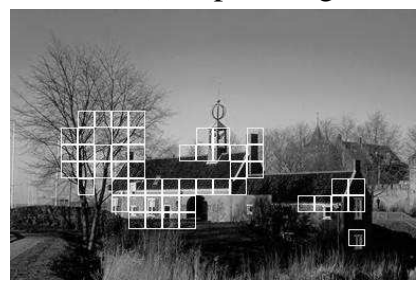

(c) MRF

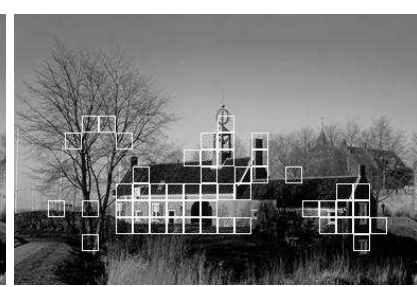

(b) Logistic

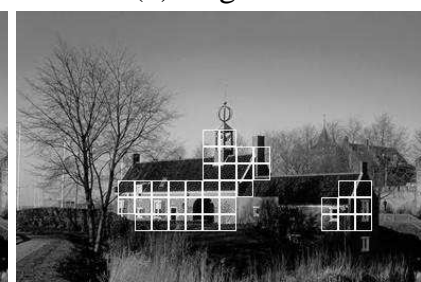

(d) DRF
Figure 2. Structure detection results on a test example for different methods. For similar detection rates, DRF reduces the false positives considerably.

for the MRF and from the MAP solution of the logistic classifier for the DRF.

For an input test image given in Figure 2 (a), the structure detection results for the three methods are shown in Figure 2. The blocks identified as structured have been shown enclosed within an artificial boundary. It can be noted that for similar detection rates, the number of false positives have significantly reduced for the DRF based detection. The logistic classifier does not enforce smoothness in the labels, which led to increased false positives. However, the MRF solution shows a smoothed false positive region around the tree branches because it does not take into account the neighborhood interaction of the data. Locally, different branches may yield features similar to those from the man-made structures. In addition, the discriminative association potential and the data-dependent smoothing in the interaction potential in the DRF also affect the detection results. An another example comparing the detection rates of the MRF and the DRF is given in Figure 3. For similar false positives, the detection rate of the DRF is considerably higher. This indicates that the data interaction is important for both increasing the detection rate as well as reducing the false positives. The ICM algorithm converged in less than 5 iterations for both the DRF and the MRF. The average time taken in processing an image of size $256 \times 384$ pixels in Matlab 6.5 on a $1.5 \mathrm{GHz}$ Pentium class machine was $2.42 \mathrm{~s}$ for the DRF, $2.33 \mathrm{~s}$ for the MRF and $2.18 \mathrm{~s}$ for the logistic classifier. As expected, the DRF takes more time than the MRF due to the additional computation of data-dependent term in the interaction potential in the DRF.

To carry out the quantitative evaluation of our work, we compared the detection rates, and the number of false positives per image for each technique. To avoid the confusion 


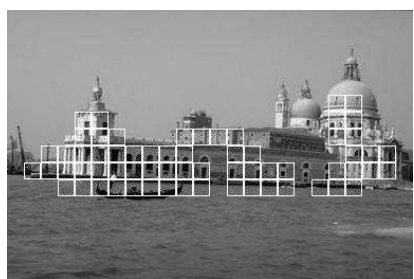

(a) MRF

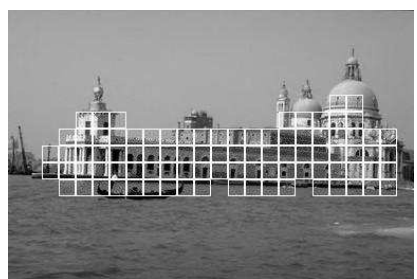

(b) DRF
Figure 3. Another example of structure detection. Detection rate of DRF is higher than that of MRF for similar false positives.
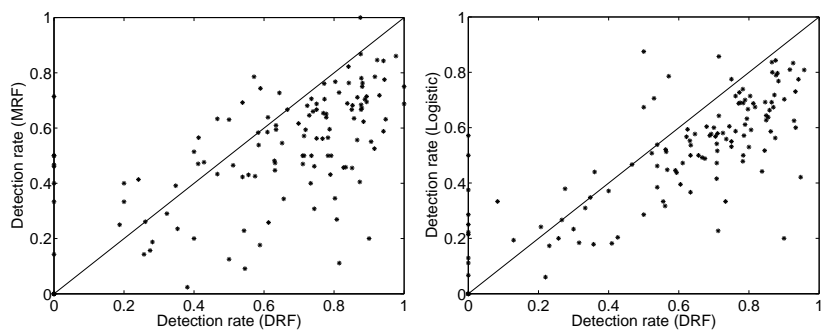

Figure 4. Comparison of the detection rates per image for the DRF and the other two methods for similar false positive rates. For most of the images in the test set, DRF detection rate is higher than others.

due to different effects in the DRF model, the first set of experiments was conducted using the single-site features for all the three methods. Thus, no neighborhood data interaction was used for both the logistic classifier and the DRF, i.e. $f_{i}=s_{i}$. The comparative results for the three methods are given in Table 1 next to 'MRF', 'Logistic ${ }^{-}$, and 'DRF' ${ }^{-}$. For comparison purposes, the false positive rate of the logistic classifier was fixed to be the same as the DRF in all the experiments. It can be noted that for similar false positives, the detection rates of the MRF and the DRF are higher than the logistic classifier due to the label interaction. However, higher detection rate of the DRF in comparison to the MRF indicates the gain due to the use of discriminative models in the association and interaction potentials in the DRF.

In the next experiment, to take advantage of the power of the DRF framework, data interaction was allowed for both the logistic classifier as well as the DRF. Further, to decouple the effect of the data-dependent term from the dataindependent term in the interaction potential in the DRF, the weighting parameter $K$ was set to 0 . Thus, only datadependent smoothing was used for the DRF. The DRF parameters were learned for this setting (Section 3 ) and $\beta$ was found to be 1.26. The DRF results (' $\mathrm{DRF}(K=0)$ ' in Table 1) show significantly higher detection rate than that from the logistic and the MRF classifiers. At the same time, the DRF reduces false positives from the MRF by more than $48 \%$.
Table 1. Detection Rates (DR) and False Positives (FP) for the test set containing 129 images. FP for logistic classifier were kept to be the same as for DRF for DR comparison. Superscript ${ }^{\prime}{ }^{\prime}{ }^{\prime}$ indicates no neighborhood data interaction was used. $K=0$ indicates the absence of the data-independent term in the interaction potential in DRF.

\begin{tabular}{|r|cc|}
\hline Method & FP (per image) & DR (\%) \\
\hline \hline MRF & 2.36 & 57.2 \\
\hline Logistic $^{-}$ & 2.24 & 45.5 \\
\hline DRF $^{-}$ & 2.24 & 60.9 \\
\hline \hline Logistic & 1.37 & 55.4 \\
\hline DRF $(K=0)$ & 1.21 & 68.6 \\
\hline DRF & 1.37 & 70.5 \\
\hline
\end{tabular}

Table 2. Results with linear classifiers (See text for more).

\begin{tabular}{|r|cc|}
\hline Method & FP (per image) & DR (\%) \\
\hline \hline Logistic(linear) & 2.04 & 55.0 \\
\hline DRF (linear) & 2.04 & 62.3 \\
\hline
\end{tabular}

Finally, allowing all the components of the DRF to act together, the detection rate further increases with a marginal increase in false positives ('DRF' in Table 1). However, observe that for the full DRF, the learned value of $K(0.83)$ signifies that the data-independent term dominates in the interaction potential. This indicates that there is some redundancy in the smoothing effects produced by the two different terms in the interaction potential. This is not surprising because the neighboring sites usually have 'similar' data. We are currently exploring other forms of the interaction potential that can combine these two terms without duplicating their smoothing effects. To compare per image performance of the DRF with the MRF and the logistic classifier, scatter plots were obtained for the detection rates for each image (Figure 4). Each point on a plot is an image from the test set. These plots indicate that for a majority of the images the DRF has higher detection rate than the other two methods.

To analyze the performance of the MAP inference for the DRF, a MAP solution was obtained using the min-cut algorithm. The overall detection rate was found to be $24.3 \%$ for 0.41 false positives per image. Very low detection rate along with low false positives indicates that MAP prefers oversmoothed solutions in the present setting. This is because the pseudolikelihood approximation used in this work for learning the parameters tends to overestimate the interaction parameter $\beta$. Our MAP results match the observations made by Greig et al. [9], and Fox and Nicholls [6] for large values of $\beta$ in MRFs. In contrast, ICM is more resilient to the errors in parameter estimation and performs well even 
for large $\beta$, which is consistent with the results of [9], [6], and Besag [1]. For MAP to perform well, a better parameter learning procedure than using a factored approximation of the likelihood will be helpful. In addition, one may also need to impose a prior that favors small values of $\beta$. We intend to explore these issues in greater detail in the future.

One of the further aspects of the DRF model is the use of general kernel mappings to increase the classification accuracy. To assess the sensitivity to the choice of kernel, we changed the quadratic functions used in the DRF experiments to compute $\boldsymbol{h}_{i}(\boldsymbol{y})$ to one-to-one transform such that $\boldsymbol{h}_{i}(\boldsymbol{y})=\left[1 \boldsymbol{f}_{i}(\boldsymbol{y})\right]$. This transform will induce a linear decision boundary in the feature space. The DRF results with quadratic boundary (Table 1) indicate higher detection rate and lower false positives in comparison to the linear boundary (Table 2). This shows that with more complex decision boundaries one may hope to do better. However, since the number of parameters for a general kernel mapping is of the order of the number of data points, one will need some method to induce sparseness to avoid overfitting [5].

\section{Conclusions}

In this work, we have proposed discriminative random fields for the classification of image regions while allowing neighborhood interactions in the labels as well as the observed data without making any model approximations. The DRFs provide a principled approach to combine local discriminative classifiers that allow the use of arbitrary, overlapping features, with smoothing over the label field. The results on the real-world images validate the advantages of the DRF model. The DRFs can be applied to several other tasks, e.g. classification of textures for which the consideration of data dependency is crucial. The next step is to extend the model to accommodate multiclass classification problems. In the future, we also intend to explore different ways of robust learning of the DRF parameters so that more complex kernel classifiers could be used in the DRF framework.

\section{Acknowledgments}

Our thanks to J. Lafferty and J. August for very helpful discussions, and V. Kolmogorov for the min-cut code.

\section{References}

[1] J. Besag. On the statistical analysis of dirty pictures. Journal of Royal Statistical Soc., B-48:259-302, 1986.

[2] H. Cheng and C. A. Bouman. Multiscale bayesian segmentation using a trainable context model. IEEE Trans. on Image Processing, 10(4):511-525, 2001.

[3] W. J. Christmas, J. Kittler, and M. Petrou. Structural matching in computer vision using probabilistic relaxation. IEEE Trans. Pattern Anal. Machine Intell., 17(8):749-764, 1995.
[4] X. Feng, C. K. I. Williams, and S. N. Felderhof. Combining belief networks and neural networks for scene segmentation. IEEE Trans. PAMI, 24(4):467-483, 2002.

[5] M. A. T. Figueiredo and A. K. Jain. Bayesian learning of sparse classifi ers. In Proc. IEEE Int. Conference on Computer Vision and Pattern Recognition, 1:35-41, 2001.

[6] C. Fox and G. Nicholls. Exact map states and expectations from perfect sampling: Greig, porteous and seheult revisited. In Proc. Twentieth Int. Workshop on Bayesian Inference and Maximum Entropy Methods in Sci. and Eng., 2000.

[7] S. Geman and D. Geman. Stochastic relaxation, gibbs distribution and the bayesian restoration of images. IEEE Trans. on Patt. Anal. Mach. Intelli., 6:721-741, 1984.

[8] P. E. Gill, W. Murray, and M. H. Wright. Practical Optimization. Academic Press, San Diego, 1981.

[9] D. M. Greig, B. T. Porteous, and A. H. Seheult. Exact maximum a posteriori estimation for binary images. Journal of Royal Statis. Soc., 51(2):271-279, 1989.

[10] J. Kittler and E. R. Hancock. Combining evidence in probabilistic relaxation. Int. Jour. Pattern Recog. Artifi cial Intelli., 3(1):29-51, 1989.

[11] J. Kittler and D. Pairman. Contextual pattern recognition applied to cloud detection and identifi cation. IEEE Trans.on Geo. and Remote Sensing, 23(6):855-863, 1985.

[12] V. Kolmogorov and R. Zabih. What energy functions can be minimized via graph cuts. In Proc. European Conf. on Computer Vision, 3:65-81, 2002.

[13] S. Kumar and M. Hebert. Man-made structure detection in natural images using a causal multiscale random fi eld. In Proc. IEEE Int. Conf. on CVPR, 1:119-126, 2003.

[14] J. Lafferty, A. McCallum, and F. Pereira. Conditional random fi elds: Probabilistic models for segmenting and labeling sequence data. In Proc. ICML, 2001.

[15] S. Z. Li. Markov Random Field Modeling in Image Analysis. Springer-Verlag, Tokyo, 2001.

[16] P. McCullagh and J. A. Nelder. Generalised Linear Models. Chapman and Hall, London, 1987.

[17] T. P. Minka. Algorithms for Maximum-Likelihood Logistic Regression. Statistics Tech Report 758, Carnegie Mellon University, 2001.

[18] W. Pieczynski and A. N. Tebbache. Pairwise markov random fi elds and its application in textured images segmentation. In Proc. 4th IEEE Southwest Symposium on Image Analysis and Interpretation, pages 106-110, 2000.

[19] Y. D. Rubinstein and T. Hastie. Discriminative vs informative learning. In Proc. Third Int. Conf. on Knowledge Discovery and Data Mining, pages 49-53, 1997.

[20] R. Wilson and C. T. Li. A class of discrete multiresolution random fi elds and its application to image segmentation. IEEE Trans. PAMI, 25(1):42-56, 2003.

[21] C. S. Won and H. Derin. Unsupervised segmentation of noisy and textured images using markov random fields. CVGIP, 54:308-328, 1992.

[22] G. Xiao, M. Brady, J. A. Noble, and Y. Zhang. Segmentation of ultrasound b-mode images with intensity inhomogeneity correction. IEEE Trans. Med. Imaging, 21(1):48-57, 2002. 